
matérielle de l'éducation

\title{
Avant-propos
}

\section{Sylvain Wagnon}

\section{(2) OpenEdition}

1 Journals

Electronic version

URL: http://journals.openedition.org/trema/3116

DOI: 10.4000/trema.3116

ISSN: 2107-0997

\section{Publisher}

Faculté d'Éducation de l'université de Montpellier

\section{Printed version}

Date of publication: 1 June 2014

Number of pages: $1-7$

ISBN: 1167-315X

ISSN: $1167-315 X$

Electronic reference

Sylvain Wagnon, «Avant-propos », Tréma [Online], 41 | 2014, Online since 01 June 2014, connection on 22 September 2020. URL : http://journals.openedition.org/trema/3116; DOI : https://doi.org/10.4000/ trema.3116

This text was automatically generated on 22 September 2020 .

Trema 


\title{
Avant-propos
}

\author{
Sylvain Wagnon
}

1 Pourquoi s'intéresser au film fixe aujourd'hui ? Medium très présent dans les classes du premier et du second degré dans les années mille-neuf-cent cinquante à soixante-dix, le film fixe est un véritable "dinosaure » par rapport aux moyens audio-visuels et numériques actuels.

2 C'est peut-être là un de ses premiers attraits pour la recherche en histoire de l'éducation. En effet, son histoire est circonscrite à une périodisation relativement précise. Techniquement, le film fixe apparaît au début des années vingt en se substituant à la plaque de verre plus coûteuse et plus fragile. Son essor hors et dans l'école reste progressif jusqu'à la circulaire Barangé de 1951 qui marque un soutien financier réel de l'institution scolaire pour ce nouveau medium et support pédagogique. Les éditeurs, plus d'une cinquantaine de maisons d'éditions pendant cet "âge d'or» du film fixe saisissent tout l'intérêt de ce nouveau marché et commercialisent des dizaines de milliers de films. Finalement, c'est la diapositive puis la télévision, au milieu des années soixante, qui amorce la mort du film fixe même si ce dernier reste présent dans les classes jusqu'à la fin des années soixante-dix.

Délimité chronologiquement, le film fixe permet donc une étude contextualisée et périodisée dont plusieurs contributions de ce numéro rappellent les étapes essentielles.

3 Ensuite, l'étude du film fixe nous donne la possibilité d'un recul par rapport à la période actuelle d'introduction massive du numérique dans les classes. C'est à notre avis aussi un des avantages de l'étude historique du film fixe: établir des liens, des comparaisons avec le mouvement actuel d'intégration des supports numériques dans les apprentissages scolaires. Les demandes sociales des parents, du secteur marchand, des enseignants, des chercheurs, l'accompagnement, parfois l'anticipation de l'institution scolaire pour toutes les technologies de l'éducation font écho à ce qu'a pu connaître l'histoire du film fixe avec ses espoirs et ses désillusions.

4 Certes, "l'actualité du passé » est toujours une dérive dangereuse du point de vue historique et méthodologique. L'expérience de l'histoire est souvent hasardeuse mais débusquer dans l'histoire de similitudes avec nos questionnements actuels, des 
résonnances, des échos conduisent à une mise en perspective de problématiques actuelles.

5 Ce numéro n'entend pas découvrir le film fixe. Son étude historique a été entamée avec rigueur par les travaux antérieurs de Thierry Lefebvre, Didier Nourrisson, Christel Taillibert et Valérie Vignaux ${ }^{1} . .$. Leurs travaux restent nos références et c'est avec grand plaisir que tous, même si certains faute de temps, n'ont pu apporter leur contribution directe, ont marqué leur intérêt pour cette nouvelle publication sur un sujet qu'ils connaissent mieux que quiconque.

Nous avons donc conçu ce numéro autour de deux axes. D'une part, avec la volonté de poursuivre les travaux sur une source encore aujourd'hui peu inventoriée et qui du point de vue quantitatif est encore fortement sous-estimée. D'autre part, d'étudier ce support pédagogique comme un élément de cette histoire matérielle de l'éducation qui représente à notre avis un champ de recherche substantiel de l'histoire de l'éducation.

\section{Le film fixe, histoire d'un support pédagogique singulier?}

7 Roulés dans de petites boîtes carrées ou rondes, en carton, métal ou plastique, de formats multiples ( $8 \mathrm{~mm}, 9.5 \mathrm{~mm}, 16 \mathrm{~mm}$ et surtout $35 \mathrm{~mm}$ ), les films fixes ont l'aspect d'une pellicule photographique avec ses bords crantés et se composent de vues positives en noir et blanc, parfois avec de la couleur suivant les périodes. Les films sont composés de dessins, de photographies, de cartes et sont accompagnés la plupart du temps d'une notice ou d'un livret pédagogique.

8 Le film fixe a connu dans les années cinquante un tel essor commercial qu'il est impossible de le limiter au domaine purement scolaire. La plupart des institutions territoriales, des pouvoirs organisateurs, des ministères, des grandes collectivités locales, des grandes entreprises et des associations en tout genre ont participé à cet élan par la production de films proposés aux enseignants où la "réclame » et la publicité sont omniprésentes. L'inventaire et la cartographie des fonds constitués de films fixes restent à faire à partir de bases communes et d'un signalement national. Leur multitude et leur caractère "pluridisciplinaire» en font une source encore aujourd'hui à découvrir' ${ }^{2}$ À titre d'exemple, l'Insep (l'Institut national du sport et de l'éducation physique) ou la cinémathèque de Nice présentent des fonds de plusieurs milliers de films fixes non encore inventoriés.

9 Il convenait tout d'abord de procéder à une périodisation qui établisse les grandes césures de l'histoire singulière du film fixe. La plupart des contributions de ce numéro proposent donc une analyse historique qui répond à ce questionnement et qui place le film fixe au cœur d'enjeux non seulement pédagogiques mais aussi idéologiques et économiques.

\section{Le film fixe, source d'une histoire de la culture matérielle de l'éducation}

Si l'histoire matérielle est un axe reconnu de l'histoire culturelle ${ }^{3}$, l'histoire culturelle matérielle de l'éducation est un axe encore nouveau.

Cette histoire culturelle matérielle s'appuie sur les travaux pionniers de Roger Chartier 
sur le livre ${ }^{4}$, sur ceux de Daniel Roche $e^{5}$ mais aussi de Georges Vigarello sur le mobilier scolaire $^{6}$.

Néanmoins, en ce qui concerne l'éducation, il nous semble que deux axes sont perceptibles. D'une part, une réflexion sur les outils scolaires et nous pensons ici particulièrement aux outils scolaires du français développé par Sylvie Plane et Bernard Schneuwly ${ }^{7}$. Ce travail pionnier ouvrait clairement un chantier de recherche sur les outils d'enseignement par leurs spécificités, leurs fonctionnements, leurs usages et leurs représentations. Pour les auteurs, les outils d'enseignement étaient ainsi un « artefact introduit dans la classe de français servant l'enseignement / apprentissage des notions et capacités ${ }^{8} »$. Le grand intérêt de ces recherches est l'ouverture de plusieurs axes de questionnements. Le premier concernant les pratiques scolaires avec l'histoire de ces outils et le second concernant le fonctionnement «réel» dans les pratiques des classes.

D'autre part, un second courant s'intéresse plus particulièrement à la culture matérielle de l'enfance et pas seulement dans le domaine scolaire. À ce titre la revue Strenae et en particulier le récent numéro coordonné par Annie Renonciat et Michel Manson soulève des pans entiers de recherches, de nouveaux territoires et de nouvelles perspectives de recherches?.

11 La description même analytique de l'histoire d'un objet ne peut suffire, il s'agit en le replaçant dans ses contextes spatiaux et temporels d'en dégager une analyse plus globale de l'histoire de l'enseignement, les sources le permettent par leur quantité et leur qualité.

12 Cette histoire s'insère aussi dans celle de la culture scolaire mise en œuvre par André Chervel ${ }^{10}$. Terme éminemment polysémique, l'approche historique de la culture scolaire, met l'accent sur l'appropriation des savoirs enseignés et des pratiques pédagogiques utilisées. Le film fixe comme source et moyen pédagogique nous apparaît comme propice à analyser ce qu'on enseigne et comment on l'enseigne. Au même titre que les textes officiels ou les manuels scolaires, avec leurs propres caractéristiques, le film fixe offre la possibilité de mettre l'accent sur cette histoire des pratiques pédagogiques en prenant en compte plusieurs interrogations : Comment et pourquoi cet outil a-t-il été introduit? Dans quels contextes techniques, économiques et idéologiques? Quels furent les soutiens et les obstacles à son introduction? Comment a-t-il été perçu par les praticiens eux-mêmes ? Quelles images sont véhiculées à travers ces films?

Du point de vue méthodologique, cet axe de recherche nécessite le croisement entre les approches historique, ethnographique et archéologique dans cette volonté d'explorer le «concret», le «matériel », le quotidien pour offrir une "relecture» ou tout au moins une nouvelle optique d'étude en Histoire de l'éducation.

14 L'écueil évident de l'histoire matérielle est de se limiter à la description de l'objet alors même que ces objets ne peuvent être compris que dans l'analyse de leurs contextes spatiaux et temporels, qui impliquent un changement d'échelle d'analyse. Si cette histoire matérielle relève de la micro-histoire, même si elle doit pouvoir participer d'une part à une macro-analyse de l'histoire de l'enseignement et d'autre part posséder une visée praxéologique en particulier pour la formation des enseignants.

15 Pour l'éducation, cette histoire matérielle est un moyen particulièrement indiqué pour « décrypter » une partie de cette « boite noire » de l'histoire de l'enseignement. 
16 Nous voudrions nous situer dans la lignée des réflexions de Daniel Roche en ce qui concerne le domaine de l'histoire de l'enseignement :

«Si nous admettons que le monde extérieur des objets n'est pas le lieu de notre totale aliénation mais le moyen d'un processus créatif, et que le rapport des individus au social passe par l'objectivation, l'histoire de consommations permet de mieux comprendre la continuité du matériel et du symbolique, l'effort d'intelligence et de travail cristallisé qui se conserve dans le moindre objet, l'union des représentations et de réalités ${ }^{11}$ ».

17 Ainsi, en complément des histoires des manuels scolaires ou de l'architecture scolaire, l'histoire d'objets didactiques, comme le film fixe, s'inscrivent dans ce courant. À côté de ces quelques questions qui sont loin d'être exhaustives, nous analyserons en quoi l'objet didactique peut être ou non à l'origine d'une culture matérielle de l'école et audelà, de la construction d'une culture scolaire avec ses innovations et ses stéréotypes.

\section{La prise en compte de l'histoire des pratiques pédagogiques}

Une histoire des pratiques pédagogiques doit donc être une évidence dans cette volonté de comprendre l'histoire de l'enseignement et des apprentissages; ce n'est pourtant pas encore le cas. L'histoire des pratiques pédagogiques pose simultanément la question de l'espace-classe, du quotidien de cette classe et des outils de ce territoire scolaire spécifique.

19 Antoine Prost en 1984 soulignait :

«La légitimité même d'une approche positive des faits d'enseignement n'est pas généralement reconnue. Cette attitude archaïque permet de transformer le débat pédagogique en débat d'idées quand il devrait être nourri d'expériences et de recherches ${ }^{12}$ ».

20 S'intéresser aux pratiques pédagogiques ne signifie pas pour nous s'intéresser seulement à une autre facette de l'histoire de l'enseignement, c'est participer à un travail historique rigoureux. Ainsi, il ne s'agit pas de privilégier de façon arbitraire l'histoire des pratiques plutôt que l'histoire des idées, ni de tenter un équilibre ou une symbiose entre les deux approches. Certes, l'histoire des pratiques pédagogiques est une histoire indiciaire et les sources manquent ou plutôt sont difficiles d'accès, car elles existent, et le film fixe en est un exemple.

21 Dans une optique historique, l'étude de la tension permanente entre théorie et pratique nécessite la connaissance des pratiques, des outils scolaires et de leur usage. Il ne s'agit évidemment pas de définir l'histoire des pratiques pédagogiques comme la seule voie pour la connaissance historique de l'acte éducatif, en sous-estimant l'importance de l'histoire des idées pédagogiques.

23 L'histoire des pratiques pédagogiques ne peut être isolée. L'histoire des idées pédagogiques et celle des pratiques pédagogiques ne sont pas deux éléments distincts mais bien dans notre esprit les deux facettes d'une même histoire à construire. 


\section{Films fixes, manuels scolaires et numérique}

24 L'étude historique du film fixe implique aussi une comparaison avec les autres supports pédagogiques et en particulier le manuel scolaire. Ensemble, il forme un "dispositif " éditorial et pédagogique.

Bien avant l'introduction du numérique, les manuels scolaires ont été associés à d'autres supports d'apprentissage qu'il s'agisse des dictionnaires, des cartes et des films fixes $^{13}$. Ainsi, il nous a semblé intéressant, en étudiant l'histoire d'un ancêtre du numérique, d'éclairer, peut-être sous un autre jour la question de « l'entrée de l'école dans l'ère du numérique ${ }^{14}$ » et sur « la possibilité d'enseigner autrement ${ }^{15}$ ».

Bien évidemment, nous n'avons pas cherché un parallélisme entre film fixe et numérique. Le film fixe reste un outil rudimentaire par rapport au numérique mais on perçoit bien que toute une série de questionnements et de problématiques restent d'actualité.

Car l'introduction du film fixe, comme celle du numérique, interroge les finalités de l'école et de toute une série de questions sur l'évolution des programmes et des contextes d'enseignement. Autrement dit, l'histoire du film fixe nous semble, sans donner de leçons de l'histoire, poser l'importance de trois éléments : la formation des enseignants, l'articulation entre la recherche et l'enseignement et le choix des méthodes pédagogiques.

C'est pour cela que l'ensemble des articles de ce numéro esquissent, à partir de grilles de lectures différentes, plusieurs facettes du kaléidoscope que représente l'univers du film fixe, objet d'étude et de recherche d'une histoire matérielle de l'éducation en construction.

\section{NOTES}

1. Nourrisson, D., un fonds éducatif réinventé, sociétés et représentations, n³1, 2011, pp. 177-188; Lefebvre, T., «Sage comme une image. L'abbé Songaylo et le Pathéorama, 1895, n²1, décembre 1997 ; Lefebvre, T., «Film safety, formats réduits, films fixes », in Nourrisson, D. et Jeunet, P. (dir.), Cinéma-École: aller-retour, Saint-Étienne, Presses Universitaires de SaintEtienne, 2001 ; Lefebvre, T., «Films fixes et santé publique », Revue d'histoire de la pharmacie, $\mathrm{n}^{\circ} 331$, 3e trimestre 2001. Nourrisson, D., « Le 7e art d'enseigner...: le film fixe », in Nourrisson, D., et Jeunet, P. (dir.), Cinéma-École : aller-retour, Saint-Étienne, Presses Universitaires de SaintÉtienne, 2001 ; Nourrisson, D., « Arrêt sur les images de l'alcoolisme ", in Nourrisson, D., (dir.), À votre santé ! Éducation et santé sous la 4e République, Saint-Étienne, Presses Universitaires de Saint-Étienne, 2002 ; Vignaux, V., « Le film fixe Pathéorama (1921) phénomène ou paradigme ?», in Lugon, O. et Guido, L. (dir.), Fixe/Animé, Lausanne, L'Âge d'Homme, 2010 ; Vignaux, V., Jean Benoit-Lévy ou le corps comme utopie, une histoire du cinéma éducateur dans l'entre-deuxguerres en France, Paris, AFRHC, 2007 ; Taillibert, C., « L'usage mixte de l'image fixe et de l'image animée dans le domaine de l'enseignement durant l'entre-deux guerres » dans Lugon, $\mathrm{O}$. et 
Guido, L., Fixe/Animé : croisements de la photographie et duc cinéma au XXe siècle, coll. « Histoire et théorie du cinéma ", L’âge d'homme, Lausanne, 2010, pp. 145-155.

2. Goutanier, C. et Lepage, J. Le film fixe : une source à découvrir, Histoire@Politique, $n^{\circ} 1,2008$.

3. Pesez, J.-M. (1978). Histoire de la culture matérielle, LeGoff, J. (dir.), La Nouvelle Histoire, Paris : Éditions Retz, 1978, p. 98-130 ; Julien, M.-P. et Rosselin, C. (2005). La culture matérielle. Paris : Maspero.

4. Chartier, R. (dir.). (1997). Histoire de la lecture dans le monde occidental. Paris : Éditions du Seuil.

5. Roche, D. (1997). Histoire des choses banales. Naissance de la consommation dans les sociétés traditionnelles (XVIIe-XIXe siècles), Paris : Fayard ; Roche, D. (1998). Le peuple de Paris, essai sur la culture populaire au XVIIIe siècle. Paris : Fayard ; Roche, D. (1989). La culture des apparences. Une histoire du vêtement, XVIIe-XVIIIe siècles. Paris : Fayard.

6. Vigarello, G. (1978), Le corps redressé, Paris : Armand Colin.

7. Plane, S. et Schneuwly, B. (dir.). (2000). Les outils d'enseignements du français. Repères. $n^{\circ} 22$.

8. Ibid.

9. Renonciat, A. et Manson, M. (dir). (2012). La culture matérielle de l'enfance : un nouvel horizon de recherche. Strenae, $n^{\circ} 4$.

10. Chervel, A. "L'histoire des disciplines scolaires : réflexions sur un domaine de recherche", Histoire de l'éducation, $\mathrm{n}^{\circ} 38$, mai 1988, pp. 59-119; Chervel, A., La Culture scolaire. Une approche historique, Paris : Belin, 1998.

11. Roche, D. (1997). Histoire des choses banales. Naissance de la consommation dans les sociétés traditionnelles (XVIIe-XIXe siècles), Paris : Fayard. p. 16.

12. Prost, A. (1984). Sur l'école. Le discours de restauration: essai d'interprétation. Le débat, n³1, septembre, p.42.

13. Journée Pierre Guibbert, Faculté d'Éducation - Université de Montpellier 2, 6 février 2014, «Les manuels scolaires et le numérique».

14. Fourgous, J.-M. (2010). Réussir l'école numérique. Rapport de la mission parlementaire sur la modernisation de l'école par le numérique. Paris : Assemblée nationale.

15. Fourgous, J.-M. (2012). Apprendre autrement à l'ère numérique. Rapport de la seconde mission parlementaire sur la modernisation de l'école par le numérique. Paris: Assemblée nationale.

\section{AUTHOR}

\section{SYLVAIN WAGNON}

Faculté d'Éducation - Université de Montpellier 2 LIRDEF 